\title{
ALTERAÇÕES AUDITIVAS E FENILCETONÚRIA: UMA REVISÃO SISTEMÁTICA
}

\section{Hearing disorders and phenylketonuria: a systematic review}

\author{
Patrícia Cotta Mancini ${ }^{(1)}$, Ana Lúcia Pimenta Starling ${ }^{(2)}$, Cláudia Fernanda Tolentino Alves ${ }^{(3)}$, \\ Thaís Maria da Mata Martins ${ }^{(4)}$, Maria Cecília Martinelli lório ${ }^{(5)}$
}

\begin{abstract}
RESUMO
Tema: a fenilcetonúria é uma doença genética que provoca alterações bioquímicas conduzindo a uma deficiência na síntese de proteínas e de neurotransmissores, e prejudicando o processo de mielinização. Mudanças estruturais e funcionais da mielina podem alterar os padrões de condutividade neuronal e ou diminuir a conexão sináptica em indivíduos com fenilcetonúria. Essencialmente, um tratamento dietético deve ser realizado dentro das primeiras semanas de vida para evitar as manifestações clínicas e bioquímicas da doença. Quando a dieta é mantida ininterruptamente, as crianças com fenilcetonúria apresentam desenvolvimento normal. Porém, foram observados déficits em funções executivas, na interação inter-hemisférica, na linguagem e memória mesmo em crianças com tratamento precoce e dieta adequada. Algumas pesquisas foram realizadas para investigação da relação entre fenilcetonúria e alterações auditivas. Objetivo: rever de forma sistemática os artigos científicos dedicados à pesquisa da relação entre alterações auditivas e hiperfenilalaninemias, destacando a fenilcetonúria clássica. As referências bibliográficas foram obtidas por meio de pesquisa nas bases de dados Lilacs, Medline, Biblioteca Cochrane e Scielo e por busca na lista de referência dos artigos identificados e selecionados. Conclusão: conclui-se que a relação entre hiperfenilalaninemias, incluindo a fenilcetonúria, e alterações auditivas ainda é controversa na literatura. Sugere-se a realização de mais investigações sobre a função auditiva nesses indivíduos a fim de elucidar essa possível relação.
\end{abstract}

DESCRITORES: Fenilcetonúrias; Transtornos da Audição; Potenciais Evocados Auditivos; Potenciais Evocados Auditivos do Tronco Encefálico

(1) Fonoaudióloga; Professora Assistente do Curso de Graduação em Fonoaudiologia da Universidade Federal de Minas Gerais, UFMG, Belo Horizonte, MG, Brasil; Doutoranda em Ciências dos Distúrbios da Comunicação Humana: Campo Fonoaudiológico da Universidade Federal de São Paulo.

(2) Médica; Professora Adjunta do Departamento de Pediatria da Faculdade de Medicina da Universidade Federal de Minas Gerais, UFMG, Belo Horizonte, MG, Brasil; Doutora em Ciências da Saúde: Saúde da Criança e do Adolescente pela Universidade Federal de Minas Gerais.

(3) Aluna do Curso de Graduação em Fonoaudiologia da Universidade Federal de Minas Gerais, UFMG, Belo Horizonte, MG, Brasil; Bolsista do Núcleo de Ações e Pesquisa em Apoio Diagnóstico.

(4) Aluna do Curso de Graduação em Fonoaudiologia pela Universidade Federal de Minas Gerais, UFMG, Belo Horizonte, MG, Brasil; Bolsista do Núcleo de Ações e Pesquisa em Apoio Diagnóstico.

(5) Fonoaudióloga; Professora Associada do Curso de Graduação em Fonoaudiologia da Universidade Federal de São Paulo, UNIFESP, São Paulo, SP, Brasil; Doutora em Ciências dos Distúrbios da Comunicação Humana: Campo Fonoaudiológico pela Universidade Federal de São Paulo.

Conflito de interesses: inexistente

\section{INTRODUÇÃO}

O nome genérico dado a elevados níveis de fenilalanina no sangue é hiperfenilalaninemia (HPA), que constitui uma desordem primária do sistema de hidroxilação da fenilalanina que pode ser causado, dentre outros fatores, pela deficiência da enzima fenilalanina hidroxilase ${ }^{1,2}$. Existem diferentes tipos de HPA que formam um grupo heterogêneo de doenças, incluindo a fenilcetonúria clássica (PKU) e variações de HPA, como a HPA persistente, a HPA branda e a PKU atípica ${ }^{1,2}$.

A PKU é a doença mais comum dentro do grupo de doenças envolvendo erros congênitos no metabolismo de aminoácidos, sendo caracterizada como uma doença genética autossômica recessiva que acarreta uma deficiência na produção da enzima fenilalanina hidroxilase ${ }^{1}$. Essa enzima é responsável pela conversão do aminoácido fenilalanina em 
tirosina, sendo esse último imprescindível para a produção de neurotransmissores ${ }^{2}$. Além disso, as células gliais ficam especialmente vulneráveis na PKU resultando em um notável comprometimento na mielinização ${ }^{3}$.

Devido ao prejuízo no processo de mielinização a PKU pode trazer graves consequências neurológicas como falhas no andar e/ou no falar, hiperatividade, tremor, microcefalia, falhas no crescimento, retardo mental, atividade autística, transtorno de conduta e déficit nas funções executivas ${ }^{4}$. Os portadores dessa doença frequentemente apresentam cabelo e pele de tonalidade claras em decorrência da deficiência na pigmentação. A falta de pigmentação, por sua vez, é ocasionada pela inibição completa da hidroxilação da tirosina pela tirosinase, que constitui a primeira etapa na formação do pigmento melanina ${ }^{2}$. Além disso, pacientes com PKU apresentam alterações na mielinização ${ }^{5}$. Em uma investigação neuropatológica em indivíduos com PKU não tratados, encontrou-se um severo retardo no desenvolvimento da mielinização e da citoarquitetura das estruturas cerebrais que apresentam maturação máxima depois do nascimento ${ }^{6}$.

A PKU ocorre em todos os grupos étnicos e sua incidência em recém-nascidos pode variar de 1:2.600 até 1:26.000, em consequência da grande variabilidade genética, sendo a média 1:10.000 2 . No Brasil, estima-se que, na cidade de São Paulo, de 1:12.000 a 1:15.000 recém-nascidos sejam portadores de PKU, e dados coletados no Rio de Janeiro revelaram uma prevalência de 1:21.766 casos de PKU ${ }^{7}$.

Quando a dieta é iniciada nas primeiras semanas de vida e mantida continuamente, os indivíduos com PKU desenvolvem-se normalmente. Entretanto, foram observados déficits nas funções executivas mesmo em crianças cujo tratamento é iniciado precocemente e mantido de forma ininterrupta ${ }^{4}$. Outros estudos apontaram déficits na interação inter-hemisférica cerebral nas crianças diagnosticadas precocemente e com dieta adequada ${ }^{8}$, além de déficits cognitivos em várias áreas (atenção, memória, linguagem, aprendizagem e funções executivas) ${ }^{9}$. Portanto, ainda são questionados os níveis plasmáticos ideais de fenilalanina a fim de evitar os efeitos neurológicos a longo prazo observados em estudos mais recentes ${ }^{2}$.

Anderson et al. ${ }^{9}$ verificaram a presença de déficits de linguagem em indivíduos com PKU diagnosticados e tratados precocemente, e sabe-se que a audição é essencial para o desenvolvimento da linguagem. Portanto, torna-se importante avaliar a audição das crianças com PKU a fim de detectar qualquer possível alteração na condução do estí- mulo auditivo ocasionado por uma desmielinização subclínica.

Por isso, o objetivo desta revisão é investigar a relação entre HPA, sobretudo a PKU, e alterações auditivas, por meio da descrição e avaliação sistemática de artigos científicos sobre esse tema.

\section{MÉTODOS}

A busca na literatura foi realizada no período de 15 de março a 10 de julho de 2008, a partir das seguintes bases de dados on-line: LILACS, MEDLINE, Biblioteca Cochrane e Scielo. Essas bases foram acessadas por meio da Biblioteca Virtual em Saúde (BVS) e da Pubmed. Outra estratégia utilizada foi a busca em listas de referências dos artigos identificados e selecionados ${ }^{10}$.

Foram utilizados os seguintes descritores: "Phenylketonurias"; "Hearing"; "Auditory Perception"; "Hearing Loss; Mixed Conductive-Sensorineural"; "Hearing Impaired Persons"; "Hearing Loss, Bilateral"; "Hearing Loss, Central"; "Hearing Loss, Sensorineural"; "Hearing Disorders"; "Hearing Tests"; "Hearing Loss, High-Frequency"; "Hearing Loss, Unilateral"; "Hearing Loss"; "Hearing Loss, Conductive"; "Hearing Loss, Sudden"; "EventRelated Potentials, P300" e "Evoked Potentials, Auditory".

Os artigos identificados pela estratégia de busca inicial foram avaliados independentemente por dois autores ${ }^{10}$, conforme os seguintes critérios de inclusão: (1) estudos com indivíduos com PKU ou outro tipo de HPA, de qualquer idade, (2) estudos que realizassem qualquer tipo de avaliação da função auditiva em pacientes com PKU ou HPA.

Não foram utilizadas escalas para avaliação metodológica dos artigos selecionados, como sugerido por Sampaio e Mancini ${ }^{10}$, devido ao pequeno número de artigos encontrados, ao restrito número de indivíduos avaliados em cada estudo, além das diferentes técnicas de avaliação auditiva utilizadas.

A busca nas bases de dados Scielo e Biblioteca Cochrane não identificou nenhuma referência seguindo as estratégias definidas. Por meio da busca em referências bibliográficas dos estudos encontrados, identificou-se um estudo que foi incluído na presente revisão. Foram identificadas publicações nos idiomas português, inglês e espanhol, sendo incluído na presente revisão um total de oito artigos.

\section{REVISÃO DA LITERATURA}

Sabe-se que a PKU é uma doença que altera a mielinização ${ }^{2,11,12}$, interferindo na formação dos processos dendríticos neuronais e consequentemente 
dificultando a transmissão dos estímulos nervosos ${ }^{11,12}$. A patogenia das desordens neurológicas associadas a esse erro inato do metabolismo é estudada até os dias atuais, e ainda restam dúvidas acerca da exata natureza das alterações encontradas na substância branca de indivíduos com PKU 3,6,11. Estudos realizados por meio de ressonância magnética nesses indivíduos revelam lesão difusa por acúmulo de metabólito hidrofílico sem, no entanto, alterar a arquitetura e estrutura da substância branca ${ }^{11,12}$.

No seu trajeto desde o nervo auditivo até o córtex cerebral o estímulo auditivo percorre uma via exclusivamente neuronal e sináptica, ativando primeiramente o nervo auditivo e, em sequência, as estruturas da ponte (núcleo coclear, corpo trapezoide e complexo olivar superior) e do tronco encefálico (lemnisco lateral) ${ }^{13}$. Sendo assim, as doenças desmielinizantes podem acarretar dificuldades na transmissão do estímulo auditivo, sendo indicado a realização dos potenciais evocados auditivos do tronco encefálico (PEATE) para identificar possíveis lesões na atividade sincronizada da via auditiva ${ }^{13}$. Sendo a PKU uma doença que acarreta alterações na mielinização, mesmo em indivíduos diagnosticados e tratados precocemente ${ }^{3,11,12}$, torna-se importante avaliar os estudos dedicados à pesquisa da relação entre alterações auditivas e PKU.

Em 1962, foram relatados dois casos nos quais os pais de crianças com PKU, ainda não diagnosticadas, suspeitavam que seus filhos eram surdos ou que apresentavam alguma dificuldade de audição. Foi realizada audiometria tonal condicionada em uma criança de dois e outra de quatro anos de idade, sendo encontrados limiares de audibilidade dentro dos padrões de normalidade nas duas crianças avaliadas ${ }^{14}$.

Em outro estudo investigou-se a relação entre performance escolar e funções cognitivas, sendo avaliada a memória auditiva e habilidades de aprendizado auditivo-verbal em 26 crianças com PKU, tratadas precocemente e comparadas com 21 crianças sem PKU. Nessa pesquisa, não foi encontrada relação significativa entre alteração na memória auditiva e problemas escolares, quando os pacientes portadores de PKU, tratados precocemente, foram comparados com o grupo controle, já que apenas um paciente do grupo de estudo apresentou alteração na memória auditiva ${ }^{15}$.

Os PEATE são potenciais auditivos de curta latência, capazes de estimar os limiares auditivos eletrofisiológicos sem a colaboração do paciente, bem como informar sobre a integridade do funcionamento das vias auditivas retrococleares ${ }^{13}$. Alguns estudos que avaliaram os parâmetros neurofisiológicos auditivos em indivíduos com HPA ou PKU evi- denciaram algumas diferenças quando foram comparados os resultados com indivíduos sem essas alterações ${ }^{5,16}$. Em um estudo no qual se investigou os PEATE em pacientes pediátricos com diferentes distúrbios neurológicos, verificou-se um aumento da latência do intervalo I-V na pesquisa desses potenciais em uma criança com PKU não tratada de 2 anos de idade ${ }^{5}$. Dado o pequeno número de dados (apenas um paciente para coleta de dados), o resultado dessa pesquisa não é expressivo.

Corroborando esse achado, um estudo longitudinal prospectivo analisou o desenvolvimento dos PEATE durante o primeiro ano de vida em oito crianças com PKU tratadas precocemente e 58 crianças sem PKU do grupo controle ${ }^{16}$. Os PEATE foram realizados aos $3,6,9$ e 12 meses de idade nas crianças com PKU. Como resultado, concluiuse que a latência média do interpico I-V foi significativamente maior no grupo de crianças com PKU quando comparadas ao grupo controle. Os três principais componentes dos PEATE (ou seja, as ondas I, III e V) estavam presentes em todas as crianças com PKU durante todo primeiro ano de vida. Nesse estudo, não foram encontradas diferenças consistentes na morfologia das ondas nas crianças com PKU quando comparadas com ondas de crianças normais. A latência da onda I foi similar ao valor encontrado no grupo controle durante todo o ano. Não foram encontradas ainda diferenças entre os parâmetros neurofisiológicos auditivos e sinais bioquímicos ou clínicos no grupo de crianças PKU tratadas precocemente quando comparadas com o grupo controle ${ }^{16}$. Apesar do número reduzido de pacientes no grupo de estudo, os autores ainda encontraram diferença estatisticamente significante da latência dos interpicos I-V.

Discordando dos achados que revelaram alterações nas latências dos interpicos I-V dos PEATE de fenilcetonúricos, outro estudo não encontrou diferença significante na latência e na forma das ondas I-V ao pesquisar os PEATE de 34 adolescentes com tipos diferentes de HPA e 27 adolescentes no grupo controle ${ }^{17}$. Entretanto, esse estudo evidenciou uma diferença interaural significante na latência dos interpicos I-V e III-V. Esses intervalos estavam maiores tanto nos pacientes com HPA tratados precocemente quanto nos tratados tardiamente quando comparados com o grupo controle. No entanto, essa diferença interaural não pôde ser observada nos pacientes com HPA tipo $3{ }^{17}$. Os autores não compararam os indivíduos tratados precoce e tardiamente. A análise estatística foi realizada comparando os sujeitos com HPA e indivíduos normais, sendo a diferença interaural da latência dos interpicos I-V e III-V estatisticamente significante. Não houve equivalência entre o 
número de indivíduos em cada grupo, sendo que a amostra contou com um número menor de indivíduos no grupo controle, o que pode ter influenciado nos resultados obtidos.

Um estudo clínico e eletrofisiológico realizou uma pesquisa de PEATE em 22 adolescentes com tipos diferentes de HPA, sendo 20 tratados precocemente e dois tratados tardiamente, e com 30 indivíduos do grupo controle ${ }^{18}$. Os autores concluíram que todos os pacientes tratados durante o primeiro mês de vida apresentaram latências absolutas das ondas e intervalos interpicos dentro dos padrões de normalidade. Não houve diferença estatística entre os pacientes tratados precocemente e o grupo controle. Entretanto, um paciente tratado tardiamente apresentou prolongamento das latências dos interpicos I-III, III-V e I-V ${ }^{18}$.

Em outro estudo foi também realizada avaliação dos PEATE em 20 fenilcetonúricos tratados precocemente e dois tratados tardiamente ${ }^{19}$. Como resultado, os autores não encontraram alterações dos PEATE em pacientes tratados precocemente, mesmo ao comparar o controle da dieta. Entretanto, um dos dois pacientes fenilcetonúricos tratados tardiamente apresentou irregularidades nos PEATE. Os autores concluíram que pode existir uma pequena irregularidade nas vias auditivas centrais em pacientes fenilcetonúricos, embora ainda não esteja esclarecida a sua relação com o controle da dieta ${ }^{19}$. Essa pesquisa apresentou um número reduzido de pacientes com PKU tratados tardiamente. Além disso, esses indivíduos não foram incluídos na análise estatística, o que torna o resultado encontrado não conclusivo.

Em síntese, foi verificado um aumento da latência do intervalo I-V em dois estudos dos PEATE: um em crianças com PKU tratadas precocemente ${ }^{16}$ e outro em uma criança com PKU não tratada ${ }^{5}$. A latência do intervalo I-V é considerada uma estimativa do tempo de transmissão nas vias auditivas periféricas e do tronco encefálico ${ }^{13}$. Em crianças normais este valor reduz desde o nascimento até o primeiro ano de vida, quando as latências se aproximam do valor dos adultos ${ }^{17}$. O decréscimo dependente da idade observado na latência dos intervalos interpicos na criança normal é atribuído ao processo de mielinização ou a mudanças na eficiência das sinapses nos vários núcleos das vias auditivas ${ }^{17}$.

Adultos com PKU tratados precocemente apresentam menores déficits e alterações neurofisiológicas ${ }^{2}$. Concordando com esse achado, em dois estudos nos quais foram pesquisados os PEATE de indivíduos com PKU, não foram encontradas alterações em pacientes tratados precocemente. Entretanto, nesses dois estudos encontraram-se irregularidades nos PEATE de pacientes com PKU tratados tardiamente ${ }^{18,19}$.

O P300 (potencial auditivo de longa latência, também conhecido como potencial cognitivo) é gerado quando há percepção, reconhecimento e integração do estímulo sonoro com outra atividade cerebral ${ }^{20}$. Além das alterações encontradas em algumas pesquisas dos PEATE em indivíduos com HPA ou PKU, um estudo aponta também alterações no P300 em pacientes com PKU tratados tanto precocemente e quanto tardiamente ${ }^{21}$. Em pesquisa realizada com 15 pacientes com PKU de 7 a 22 anos de idade (sendo 11 tratados precocemente e quatro tardiamente) e com 34 indivíduos no grupo controle de 11 a 14 anos de idade, pôde-se observar uma diferença estatisticamente significante na diminuição da amplitude das ondas e um leve atraso. Não houve, porém, significância estatística da latência no grupo de fenilcetonúricos quando comparado ao grupo controle. Enquanto o P300 foi observado em todos os sujeitos do grupo controle, constatouse sua presença em apenas nove pacientes com $\mathrm{PKU}$, sendo oito tratados precocemente e um tardiamente. O P300 foi evocado em todos os pacientes precoces com dieta controlada, contudo não foi evocado em três dos quatro pacientes precoces sem controle da dieta. Além disso, esse potencial não foi encontrado em três dos quatro indivíduos com tratamento tardio, sendo que dois indivíduos estavam com a dieta controlada e um sem controle da dieta. Ambos os resultados apresentaram significância estatística, apesar do número reduzido de pacientes com PKU pesquisados ${ }^{21}$.

\section{CONCLUSÃO}

A maioria dos autores consultados realizou estudo dos parâmetros neurofisiológicos auditivos em pacientes com HPA, incluindo a PKU. Foi encontrado somente um estudo de caso correlacionando a PKU e um possível déficit auditivo. Não foi encontrado nenhum estudo dos potenciais auditivos de média latência em indivíduos com PKU, bem como nenhum estudo no qual toda a via auditiva fosse avaliada pelos métodos neurofisiológicos disponíveis.

Alterações das latências dos PEATE estão sendo interpretadas principalmente como um efeito da desmielinização. Assim, justifica-se a importância da pesquisa desses potenciais em indivíduos com PKU pelo fato de essa ser um erro congênito do metabolismo no qual pode haver alterações na mielinização. Além disso, os métodos neurofisiológicos podem detectar e definir alterações subclínicas no sistema nervoso central e periférico, incluindo o sistema auditivo, em pacientes com PKU. 
Com base na presente revisão, verifica-se que a relação entre HPA (incluindo a PKU) e alterações auditivas ainda é controversa e pouco relatada na literatura. Enquanto alguns estudos encontraram alterações nos parâmetros neurofisiológicos auditivos em pacientes com HPA ou PKU com tratamento precoce ou tardio, outros apresentam resultados dentro dos padrões de normalidade. Tendo em vista a escassez de estudos que relacionam alterações auditivas e HPA/PKU e a maior prevalência de estudos que abordam a pesquisa de PEATE em indivíduos com HPA ou PKU, sugere-se a necessidade de investigações mais completas de toda a via auditiva nesses indivíduos a fim de elucidar a possível relação entre HPA/PKU e alterações auditivas. Identificar precocemente essas lesões possibilitaria desenvolver novas abordagens terapêuticas e recursos auxiliares que minimizariam os déficits provocados pela fenilcetonúria por meio de uma estimulação auditiva específica.

\section{AGRADECIMENTOS}

Ao NUPAD- Faculdade de Medicina da UFMG e à CAPES pelo apoio financeiro durante a elaboração dessa pesquisa.

\section{ABSTRACT}

Background: phenylketonuria is a genetic disorder that causes biochemical alterations, leading to a deficiency in the synthesis of proteins and neurotransmitters and thereby hindering the myelination process. Structural and functional changes in myelin can alter neural conductivity patterns and or reduce synaptic connection in individuals with phenylketonuria. Essentially, a dietary treatment should be implemented in the first weeks of life in order to avoid clinical and biochemical manifestations of the disease. When diet is continually maintained, children with phenylketonuria show normal development. However, deficits in executive functions, interhemispherical interaction, language and memory have been observed even in children with early treatment and appropriate diet. Some researches were carried out for investigating the relationship between phenylketonuria and alterations in the hearing function. Purpose: to systematically review articles dedicated to the research of relationship between hearing disorders and hyperphenylalaninaemias, highlighting the classic phenylketonuria. The bibliographical references were obtained through research in the databases: Lilacs, Medline, Cochrane Library and Scielo and through search in the reference list of the identified and selected articles. Conclusion: the relationship among hyperphenylalaninaemias, including phenylketonuria, and hearing alterations is still controversial in the literature. It is suggested that more investigations as for the hearing function are necessary on those individuals in order to elucidate this possible relationship.

KEYWORDS: Phenylketonurias; Hearing Disorders; Evoked Potentials, Auditory; Evoked Potentials, Auditory, Brain Stem

\section{REFERÊNCIAS}

1. Mira NVM, Marquez UML. Importância do diagnóstico e tratamento da fenilcetonúria. Rev Saúde Pública. 2000; 34(1):86-96.

2. Scriver CR, Beaudet AL, Valle D, Sly WS. The metabolic and molecular bases of inherited disease. 8. ed. New York: McGraw-Hill Inc; 2001. p. 1667-724.

3. Dyer CA, Kendler A, Philibotte T, Gardiner P, Cruz J, Levy HL. Evidence for central nervous system glial cell plasticity in phenylketonuria. J Neuropathol Exp Neurol. 1996; 55(7):795-814.

4. Malloy-Diniz LF, Cardoso-Martins C, Carneiro KC, Cerqueira MMM, Ferreira APA, Aguiar MJB, et al.
Funções executivas em crianças fenilcetonúricas: variações em relação ao nível de fenilalanina. Arq Neuropsiquiatr. 2004; 62(2B):473-9.

5. Hecox KE, Cone B, Blaw ME. Brainstem auditory evoked response in the diagnosis of pediatric neurologic diseases. Neurology. 1981; 31(7):832-40.

6. Bauman ML, Kemper TL. Morphologic and histoanatomic observations of the brain in untreated human phenylketonuria. Acta Neuropathol. 1982; 58(1):55-63.

7. Ratto K, Brasil C, Boaretto M, Gomes M, Urquiza $\mathrm{H}$. Programa de triagem neonatal na cidade do Rio de Janeiro: o teste do pezinho. In: Anais de Resumos do $6^{\circ}$ Congresso Brasileiro de Saúde 
Coletiva; 2000. Salvador (BA)/ Rio de Janeiro: ABRASCO; 2000. p. 358.

8. Banich MT, Passarotti AM, White DA, Nortz MJ, Steiner RD. Interhemispheric interaction during childhood: II. Children with early-treated phenylketonuria. Dev Neuropsychol. 2000; 18(1):53-71.

9. Anderson PJ, Wood SJ, Francis DE, Coleman L, Anderson V, Boneh A. Are neuropsychological impairments in children with early-treated phenylketonuria (PKU) related to white matter abnormalities or elevated phenylalanine levels?. Dev Neuropsychol. 2007; 32(2):619-748.

10. Sampaio RF, Mancini MC. Estudos de revisão sistemática: um guia para síntese criteriosa da evidência científica. Rev Bras Fisioter. 2007 Jan/ Fev; 11(1):83-9.

11. Brenton DP, Pietz J. Adult care in phenylketonuria and hyperphenylalaninaemia: the relevance of neurological abnormalities. Eur J Pediatr. 2000; 159(Suppl2):S114-20.

12. Leuzzi V, Tosetti M, Montanaro D, Carducci $C$, Artiola C, Antonozzi I, et al. The pathogenesis of the white matter abnormalities in phenylketonuria. A multimodal 3.0 tesla MRI and magnetic resonance spectroscopy (1H MRS) study. J Inherit Metab Dis. 2007; 30(2):209-16.

13. Munhoz MSL, Silva MLG, Caovilla HH, Frazza MM, Ganança MM, Câmera JLS. Respostas auditivas de tronco encefálico. In: Munhoz MSL, Caovilla HH, Silva MLG, Ganança MM. Audiologia clínica. São Paulo: Atheneu; 2003. p.191-220.

14. Little SW. Suspected hearing defects in phenylketonuria. Arch Otolaryngol. 1962 Jun; 75(6):515-8.
15. Gassió R, Fusté E, López-Sala A, Artuch R, Vilaseca MA, Campistol J. School performance in early and continuously treated phenylketonuria. Pediatr Neurol. 2005 Oct; 33(4):267-71.

16. Cardona F, Leuzzi V, Antonozzi I, Benedetti $P$, Loizzo A. The development of auditory and visual evoked potentials in early treated phenylketonuric children. Electroencephalogr Clin Neurophysiol. 1991Jan-Feb; 80(1):8-15.

17. Korinthenberg $R$, Ullrich $K$, Füllenkemper $F$. Evoked potentials and electroencephalography in adolecents with phenylketonuria. Neuropediatrics. 1988; 19(4):175-8.

18. Ludolph AC, Ullrich K, Nedjat S, Masur H, Bick $U$. Neurological outcome in 22 treated adolescents with hyperphenylalaninemia. A clinical and electrophysiological study. Acta Neurol Scand. 1992 Apr; 85(4):243-8.

19. Ludolph AC, Vetter U, Ullrich K. Studies of multimodal evoked potentials in treated phenylketonuria: the pattern of vulnerability. Eur $\mathrm{J}$ Pediatr. 1996; 155(Suppl 1):S64-8.

20. Munhoz MSL, Silva MLG, Ganança MM, Caovilla HH, Frazza MM. Respostas auditivas de longa latência. In: Munhoz MSL, Caovilla $\mathrm{HH}$, Silva MLG, Ganança MM. Audiologia clínica. São Paulo: Atheneu; 2003. p.231-42.

21. Leuzzi V, Seri S, Cerquiglini A, Carducci $\mathrm{Cl}$, Carducci CA, Antonozzi I. Derangement of the dopaminergic system in phenylketonuria: study of the event-related potential (P300). J Inherit Metab Dis. 2000; 23(4):317-20.
RECEBIDO EM: 15/12/2008

ACEITO EM: 21/10/2009

Endereço para correspondência:

Patrícia Cotta Mancini

Rua Goitacazes, 201 / 202

Belo Horizonte - MG

CEP: 30190-050

E-mail: patmancini@gmail.com 